\title{
Quasar X-ray spectra revisited
}

\section{Citation}

Shastri, P., B. J. Wilkes, M. Elvis, and J. McDowell. 1993. "Quasar X-Ray Spectra Revisited." The Astrophysical Journal 410 (June): 29. doi:10.1086/172721.

\section{Published Version}

doi:10.1086/172721

\section{Permanent link}

http://nrs.harvard.edu/urn-3:HUL.InstRepos:32955264

\section{Terms of Use}

This article was downloaded from Harvard University's DASH repository, and is made available under the terms and conditions applicable to Other Posted Material, as set forth at http:// nrs.harvard.edu/urn-3:HUL.InstRepos:dash.current.terms-of-use\#LAA

\section{Share Your Story}

The Harvard community has made this article openly available.

Please share how this access benefits you. Submit a story.

Accessibility 


\title{
N $93-14860$
}

\section{Quasar X-ray Spectra Revisited}

\author{
P. Shastri ${ }^{1}$, B.J. Wilkes ${ }^{2}$, M. Elvis ${ }^{2}$ and J. McDowell ${ }^{2}$ \\ May 2, 1992 \\ Ap.J. Submitted
}

1: Department of Astronomy, University of Texas, Austin

2: Harvard-Smithsonian Center for Astrophysics, Cambridge

\section{Abstract}

A sample of 45 quasars observed by the IPC on the Einstein satellite is used to re-examine the relationship between the soft $(0.2-3.5 \mathrm{keV}) \mathrm{X}$-ray energy index and radio-loudness. We find that, (1) the tendency for radio-loud quasars to have systematically flatter $\mathrm{X}$-ray slopes than radio-quiet quasars is confirmed with the soft X-ray excess having negligible effect; (2) there is a tendency for the flatness of the $\mathrm{X}$-ray slope to correlate with radio core-dominance for radio-loud quasars, suggesting that a component of the $\mathrm{X}$-ray emission is relativistically beamed; (3) for the RQQs the soft X-ray slopes, with a mean of $\sim 1.0$, are consistent with the slopes found at higher energies $(2-10 \mathrm{keV})$ although steeper than those observed for Seyfert 1 galaxies (also 2-10 keV) where the reflection model gives a good fit to the data; (4) the correlation of FeII emission line strength with X-ray energy index is confirmed for radio-quiet quasars using a subset of 18 quasars. The radio-loud quasars show no evidence for a correlation. This relation suggests a connection between the ionizing continuum and the line emission from the broad emission line region (BELR) of radio-quiet quasars, but in the opposite sense to that predicted by current photoionization models; (5) the correlations of X-ray slope with radio core dominance and FeII equivalent width within the radio-loud and radio-quiet sub-classes ree pectively imply that the observed wide range of $\mathrm{X}$-ray spectral slopes is real rather than due to the large measuring uncertainties for individual objects.

\section{Introduction}

The powerful X-ray emission that is commonly seen from quasars and other active galactic nuclei is widely believed to originate close to the "central engine" in these objects. Recent studies have made it clear that there is a wide range in the energy indices of the X-ray emission, both in the soft ( 0.3-2keV: Wilkes and Elvis 1987) and hard (2-10keV: Comastri et al. 1992; Williams et al. 1992) energy ranges, laying to rest the older view that Xray slopes of all broad-line active galactic nuclei can be adequately described by a single 
power law of energy slope $\sim 0.7$ (e.g. Mushotzky 1984). The details of the X-ray emission mechanisms in different types of quasars, however, are not well understood.

Wilkes and Elvis (1987) derived X-ray energy indices, $\alpha_{E}\left(f_{\nu} \sim \nu^{-\alpha_{B}}\right)$, in the soft X-ray region $(0.2-3.5 \mathrm{keV})$ for a sample of 33 quasars that were observed with sufficient signal-tonoise with the Einstein IPC. They reported that the best-fit power-law energy slopes have a wide range ( -0.2 to 1.8$)$, and that radio-loudness correlated with the soft $\mathrm{X}$-ray energy index in the sense that radio-loud quasars (RLQs) had flatter X-ray slopes than radio-quiet quasars (RQQs). They argued for a two-component model for the $\mathrm{X}$-ray emission: one linked to the radio-emission and of somewhat flatter slope $(\sim 0.5)$; and the second of steeper slope $(\sim 1)$ that was ubiquitous in quasars. Wilkes and Elvis (1987) explain the difference between these soft slopes and the mean slope of $\sim 0.7$ observed at higher energies in terms of a mixing of the two components. They also found evidence for an upturn in the X-ray spectrum at lower energies $(<0.3 \mathrm{keV})$ - the "ultrasoft excess" - as discovered by EXOSAT (Arnaud et al. 1985).

Here we study the behavior of the X-ray energy indices of quasars with an enlarged sample of 45 objects using new IPC spectral fits from the atlas of quasar energy distributions (Elvis et al. 1992). When required by the ultra-soft excess, we use updated X-ray slopes from Masnou et al. (1992), which have been determined with a broken power-law which properly accounts for this excess. We re-examine the connection with radio-loudness (Wilkes and Elvis 1987) and with the radio core-dominance parameter (Shastri 1991), and also consider a possible relation between X-ray slope and the strength of the FelI(optical) emission lines suggested by Wilkes, Elvis and McHardy (1987) but later questioned by Boroson (1989) and Zheng and O'Brien (1990).

\section{Radio-loud and radio-quiet quasars}

Here we define radio-loudness $\left(\mathrm{R}_{L_{t}}\right)$ as $\log \left(f_{R} / f_{B}\right)$, where $f_{R}$ and $f_{B}$ are the flux densities (in $\mathrm{mJy}$ ) in the $5 \mathrm{GHz}$ and $B$ bands respectively. $f_{B}$ is calculated from the $B$ magnitude using $\mathrm{m}_{B}(0)=4440 \mathrm{Jy}$ (Johnson 1966). When $B$ magnitudes were not available $\mathrm{V}$ magnitudes were used. Note that this definition differs from the one adopted by Wilkes and Elvis (1987) who took $f_{R}$ to be the core radio flux density where available rather than the total value. Here we use the radio-loudness parameter $\left(R_{L_{t}}\right)$ to distinguish between quasars that do exhibit the phenomenon of powerful radio jets and those that do not. This change largely affects the values for RLQs. In Fig. la we show the distribution of radio-loudness for the quasars in our sample. The distribution is clearly bimodal. A quasar is thus classified as "radio-loud" when $\log \left(f_{R} / f_{B}\right)>2$.

In Tables 1 and 2, the updated soft X-ray energy indices are listed for the RQQs and RLQs respectively. The distributions of the $\mathrm{X}$-ray energy indices are shown in Fig. 1b. The RLQs have stochastically flatter X-ray spectral slopes than the RQQs at a level $>99.95 \%$ as given by a Mann-Whitney $U$ test. This confirms the earlier result of Wilkes and Elvis (1987). The RQQ $1803+676$ remains an exceptional object with an extremely flat $(\simeq-0.2)$ slope.

A simple mean of the X-ray slopes for the RQQs is $1.0 \pm 0.1$, in good agreement with the mostly overlapping sample of Wilkes and Elvis (1987), and with the higher energy (2-10 
$\mathrm{keV}$ ) slopes determined from EXOSAT and Ginga for a similar sample of quasars (Comastri et al. 1992; Williams et al. 1992). A weighted mean is somewhat lower, $0.85 \pm 0.02$, due to the strong dominance of a small number of objects with well-determined slopes. Notably, the quasar slopes are systematically steeper than the high energy slopes seen in Seyfert 1 galaxies where reflection of X-rays from cool, optically thick gas gives a good fit to the data (Pounds et al. 1990, Williams et al. 1992).

In Fig. 2 the radio-loudness parameter $\left(R_{L_{t}}\right)$ is plotted against the soft $X$-ray energy index. The systematic difference between $R L Q s$ and $R Q Q s$ reported by Wilkes and Elvis (1987) is once again clear (see also Fig 1b). The formal significance of a correlation between $\alpha_{E}$ and $R_{L_{t}}$ is $>99.5 \%$ ) although the distribution of the data suggests two classes of objects rather than a real correlation. There is no significant correlation within the sub-samples of RQQs or RLQs (<90\% probability, Spearman Rank test), supporting the idea that they form two different populations (Peacock, Miller and Mead, 1986; Smith and Heckman 1990; Williams et al. 1992). The dichotomy is consistent with the suggestion that differing emission mechanisms dominate the X-ray emission in the two classes, as originally suggested by Zamorani et al. (1981).

\section{X-ray slopes and radio-core dominance}

In the framework of the "unified interpretation" for RLQs (Blandford and Konigl 1979), bulk relativistic motion is ubiquitous in them. The observed flux density of the nuclear component is then Doppler-boosted at small angles of the motion to the line of sight, and differences between radio core-dominated (CDQs) and lobe-dominated quasars (LDQs) are due to orientation alone. The core-dominance parameter, $R$ (the ratio of the radio flux density from the nucleus to that from the radio lobes) is then a statistical measure of the angle of inclination of the nuclear jet to the line of sight (Kapahi and Saikia 1982; Orr and Browne 1982). Interpreting correlations of $X$-ray luminosity with nuclear and lobe radio luminosities in this framework, Browne and Murphy (1987) suggested that a component of the X-ray emission in RLQs is orientation dependent.

The soft X-ray slopes have been found to be systematically flatter for core-dominated quasars than for lobe-dominated quasars; (Canizares and White 1989; Boroson 1989; Shastri 1991). Based on this trend, Shastri (1991) suggested that the "radio-linked" component of the X-ray emission (Zamorani et al. 1981; Kembhavi et al. 1986; Wilkes and Elvis 1987) is probably relativistically beamed.

The values of the core-dominance parameter $(R)$ determined at an observed frequency of $5 \mathrm{GHz}$ for the RLQs in our sample are given in Table 2. Fig. 3 shows that the X-ray energy index for our larger sample correlates inversely with $\log R$, except for two quasars that are highly discrepant (sc-below). A Spearman rank test shows the correlation to be significant at the $99.95 \%$ level (one-tailed test) without the discrepant objects, and at the $90 \%$ level if they are included. Note that we have excluded the one Compact Steep Spectrum quasar in the sample (3C 48:0134+329) from Fig. 3, because, in these objects the cores often show complex radio structure (Fanti et al. 1990) and interaction of the radio jet with the interstellar medium is believed to be significant (van Breugel et al. 1984). The brightness of the nuclear components must reflect this interaction, in which case the parameter $R$ is not 
a good measure of orientation.

In the framework of the unified interpretation, the correlation in Fig. 3 is either due to a flat-spectrum $\mathrm{X}$-ray component dominating at face-on inclinations, or due to a steepspectrum component dominating at edge-on inclinations. It certainly cannot be explained by the anisotropy of the steep "ultrasoft excess" (Wilkes and Elvis 1987), since such an anisotropy would go in the opposite sense if the emission is from a central disc as has been suggested, besides dominating in a lower energy range. On the other hand, as was argued in Shastri (1991), bulk relativistic motion of material emitting a flat-spectrum X-ray component, (such as the nuclear jet), would give the required anisotropy. This suggestion fits in with the picture of an additional, flat-spectrum $\mathrm{X}$-ray component associated with the radio emission in RLQs (Zamorani et al. 1981; Kembhavi et al. 1986; Wilkes and Elvis 1987). X-ray emission from radio jets is not unknown. Burns et al. (1983) find very good spatial coincidence of structures of the jet in Centaurus $A$ as observed in the radio and Xray bands. Harris and Stern (1987) measure a position for the candidate $\mathrm{X}$-ray jet feature in $3 \mathrm{C} 273$ which is coincident with one of the optical jet features imaged by Fraix-Burnet and Nieto (1988) (the latter's feature B). Similar coincidence is reported for knots in M87 (Biretta, Stern and Harris 1991).

The above correlation with $\mathrm{R}$ would contribute to the correlation of $\mathrm{X}$-ray slope with core radio-loudness found by Wilkes and Elvis (1987), since it turns out that the core radio-loudness is correlated with $R$ for the $R L Q s$ in the sample.

The correlation in Fig. 3 is consistent with the inhomogeneous jet model of Ghisellini, Maraschi and Treves (1985), proposed for the non-thermal emission from BL Lac-type objects (also see Maraschi 1992). The model proposes two components of X-ray emission from the jet; the first is a synchrotron component from nearer the nucleus which has a steep spectrum, and is relativistically beamed but into a relatively wide angle. The second is an inverse Compton component from the outer regions of the jet, that has a flat spectrum and is beamed into a cone narrower than that of the first component, either because of higher bulk velocities or greater collimation in the outer regions of the jet. Thus the model predicts that the compound $\mathrm{X}$-ray spectrum will flatten as orientation changes from more edge-on to face-on inclinations of the nuclear jet. In the unified scheme, this would translate to flattening of the observed $\mathrm{X}$-ray spectrum with increase of radio core dominance, as seen in Fig. 3.

The trend in Fig. 3 is inconsistent with the scenario of Melia and Konigl (1989) within the unified scheme. In the context of explaining BL Lac-type objects they proposed that the hard X-ray component is due to the inverse Compton "drag" on the relativistic jet by the thermal radiation from the nuclear disc near the nucleus, and the steep spectrum component is direct synchrotron emission from further out in the jet. The hard component is more isotropic than the steep spectrum component. It follows that the steep spectrum component would then dominate at face-on orientations, and the unified scheme would predict that the steep $\mathrm{X}$-ray component dominate at large $\mathrm{R}$ values, opposite to that seen in Fig. 3 .

Tests in the framework of the unified interpretation are most meaningful when the RLQs are chosen to have a narrow spread in an orientation-independent parameter. A study to test the above result with a sample of RLQs matched with respect to their radio lobe luminosities is underway with ROSAT. We note however that no trend of X-ray slope with radio lobe luminosity is indicated for the present sample. 
It is suggestive that two of the quasars with amongst the lowest $R$ values have nominally flat $\mathrm{X}$-ray slopes (although with large uncertainties). We speculate that at the most edgeon inclinations of the quasar he steep-spectrum X-ray component may be occulted (by an obscuring torus?), leaving the residual flat-spectrum component that originates from further out in the jet still visible.

\section{X-ray spectral slopes and the optical FeII emission}

Wilkes, Elvis and McHardy (1987) suggested a correlation between the equivalent width of the FeII $\lambda 4570$ emission line strength and the soft $\mathrm{X}$-ray slope for 9 quasars. This was later disputed, however, by Boroson (1989) and Zheng and O'Brien (1990), after finding no correlation for samples of 15 and 33 quasars respectively. Boroson (1989) claimed that the inconsistency with Wilkes, Elvis and McHardy (1987) was due to a combination of samples being different and measurements being inconsistent, while Zheng and O'Brien (1990) claimed that it was their equivalent width measurements of 3 additional quasars that destroyed the correlation.

Here we present our measured FeII $\lambda 4570$ emission line strengths for 18 of the present sample of quasars. Optical spectrophotometry was obtained for all the quasars on the Multiple Mirror Telescope during the years 1985-1988. Full observational details are given in Elvis et al. (1992). The strength of the Fell $\lambda 4570$ blend was measured above a linear continuum between the continuum region immediately longwards of $\mathrm{H} \gamma \lambda 4340$ and shortwards of $\mathrm{H} \beta \lambda 4861$. The HeII $\lambda 4686$ emission line is included in this measurement, unless noted, since deblending is highly inaccurate for such heavily blended lines. HeII is a high ionization line, probably from different gas than FeII, and thus its inclusion is likely only to add scatter into any relation followed by the FeII. We note that, if the emission line widths are $>6000 \mathrm{~km} \mathrm{~s}^{-1}$, the continuum level estimate is likely to be high as the hydrogen line wings blend into those of the FeII emission (Wills 1988). Our sample was thus selected to include only quasars with lines narrower than $6000 \mathrm{~km} \mathrm{~s}^{-1}$ (with the exception of PG1613+658 where the continuum level is clear despite the large FWHM of $7050 \mathrm{~km} \mathrm{~s}^{-1}$ ). The rest frame equivalent widths as derived from our measurements are listed in Table 1 for the RQQs and Table 2 for the RLQs.

Fig. 4a shows the FeII equivalent width plotted against the soft $\mathrm{X}$-ray energy index. RQQs, CDQs ( $R>0.5)$ and LDQs $(R \leq 0.5)$ are marked separately. The correlation between Fell strength and $\alpha_{E}$ is significant at the $99 \%$ level (Spearman rank test) for the full sample or for RQQs alone, although it is clear from the figure that the RLQs show no trend. PG1416-129 has a very flat spectral index in Ginga observations (0.05 \pm 0.15 , Turner et al. 1989). Given the poor constraints on the Einstein value $(0.9 \pm 0.5,68 \%$ confidence range), the two results are consistent (the difference being $<2 \sigma$ ). If the Ginga value is used in preference (indicated by a filled square in Fig. 4a), the correlation is further strengthened ( significance $>99.5 \%$ ).

The same scatter plot with FeII $\lambda 4570$ equivalent width data from Zheng and O'Brien (1990) shows a very similar trend (Fig. 4b). There is no significant correlation for the sample as a whole (as indeed is asserted by the authors), nor for the RLQs, but the RQQs show a trend similar to Fig. 4a (significance $>95 \%$ ). There appear to be no major inconsistencies 
between their measurements and estimation procedure and ours. However, there remains one highly discrepant RQQ, 2130+099 (also in the sample of Boroson 1989, but not in our sample) which has strong FeII emission but an intermediate soft $\mathrm{X}$-ray slope (0.8).

The data of Boroson (1989) do not show a correlation even for the RQQs. He uses the equivalent width of a different FeII multiplet $(\lambda \lambda 5105-5395) \AA$, but claims that his results cannot be different as a result of this, since there is a tight correlation between the FeII $\lambda \lambda$ 5105-5395 $\AA$ and FeII $\lambda \lambda 4500-4680 \AA$ equivalent widths. 14 of Boroson's objects have data in either Zheng and O'Brien (1990) or in Tables $1 / 2$, or both. Of these, for 7 objects, the equivalent width measurements are broadly consistent with the present measurements and/or those of Zheng and O'Brien (1990); for the remaining 7, however, (including 4 RQQs), the equivalent widths of Boroson (1989) are highly discrepant. The reasons for this discrepancy are unclear. Possibilities include low signal-to-noise spectra causing large errors in the measurement, and variability.

While the high energy X-ray energy indices determined by Ginga do not by themselves show any significant correlation with the FeII equivalent width (Williams et al. 1992), the data for RQQs (available for 5 objects) are broadly consistent with Fig. 4a.

Standard photoionization models for the line emission from the Broad Line Regions of quasars (Krolik 1988) imply a close link between the spectral index of the ionizing X-ray continuum and the strength of the Fe emission lines. In the case of the RLQs, however, if the soft X-ray emission has a significant contribution from the jet as has been discussed above, then the X-ray energy index would not be a good indicator of the spectrum of the ionizing continuum. The absence of a correlation between the line strengths and the X-ray slope for RLQs would therefore be expected. Although there is a correlation for the RQQs, it is in the opposite sense to that predicted by the standard model in which the FeII emission is generated deep within the cloud and thus is sensitive to harder X-rays. It should be kept in mind though, that the standard models fail to predict the large strength of the optical FeII emission lines. Also, Zheng and O'Brien (1990) find a strong relation between the strength of the optical FeII emission and the H $\beta$ FWHM. They compare their data with the simulations of Wills (1988) and conclude that the correlation cannot be explained by overestimation of the continuum alone. They suggest that the geometry of the emitting regions may also play a role in determining the observed FeIl equivalent widths as discussed by Netzer, Laor and Gondhalekar (1992) and references therein. It is possible that geometry plays a role in the observed X-ray energy index as well, just as it appears to do in the RLQs. No correlation between $\mathrm{X}$-ray slope and $\mathrm{H} \beta$ FWHM is seen in the current sample although, as noted above, the full range of FWHM is not included here to avoid measurement problems.

\section{The range of X-ray slopes}

The existence of correlations of the X-ray spectral index with external parameters in both the radio-quiet and radio-loud sub-samples allows us to strengthen the significance of the IPC measurements.

In the case of the RQQs the equivalent widths of the FeII lines are entirely independent of the X-ray measurement so the correlation of Fig. 4a can exist only if the soft X-ray slopes have a real intrinsic range of slopes from $\sim 0$ to $\sim 1.5$. This truly is a large range. It is, for 
example, $\pm 4 \sigma$ either side of the HEAO-2/EXOSAT mean, given the dispersion measured in those samples (Petre et al. 1984, Turner and Pounds 1989). Since our sample is small and incomplete, we cannot determine the real distribution of the slopes; the extremes we observe, however, cannot be rare.

Some active galactic nuclei have been discovered with the optical spectrum completely dominated by FeII emission (Lawrence et al. 1988). Soft X-ray observations of these AGN will test the FeII/soft-X-ray-slope relation in extremis, and may uncover some of the softest AGN. The alternative possibility is to measure Fell strengths in AGN selected to have particularly soft spectra, e.g., those in the 'Ultra-Soft Survey' of Cordova et al. (1989).

For the radio-loud sample a slightly smaller range of soft $X$-ray spectral index is required, $\sim 0-1$, to produce the observed correlation of $\mathrm{X}$-ray slope and core dominance. This correlation is immediately subject to interpretation in the beaming hypothesis, as discussed above.

A wide spread of soft X-ray spectral indices for RQQs and RLQs is clearly required by their correlation with extrinsic parameters which themselves show extended ranges. For the RQQs, this implies the existence of (at least) another variable which affects their X-ray properties. This variable is at present unknown.

\section{Conclusions}

Using updated soft $X$-ray slopes for a sample of 45 quasars, we conclude the following:

- The earlier trend that radio-loud quasars have systematically flatter power-law fits to the soft $\mathrm{X}$-ray spectrum than radio-quiet quasars is confirmed.

- The lack of a significant trend when each class is considered separately implies that they belong to two separate populations.

- For the radio-quiet quasars, soft (0.2-3.5 keV) X-ray slopes are consistent with those at higher (2-10 keV) energies for radio-quiet quasars but steeper than the high energy slopes of Seyfert 1 galaxies where reflection of the $\mathrm{X}$-rays gives a good fit to the data.

- For the radio-loud quasars, X-ray slope correlates with the radio-core dominance. Within the framework of the unified interpretation this result suggests that a flat spectrum X-ray component dominates due to Doppler enhancement when the quasar jet is pointed towards us.

- A significant correlation exists between the FeII optical emission line strengths and Xray spectral index for 11 radio-quiet quasars from our sample. Radio-loud quasars do not follow the correlation, consistent with the predominance of an extra X-ray component associated with the radio jet. The correlation demonstrates a close observational link between quasar emission lines and the observed X-ray continuum which is thought to excite them. However, most current photoionization models predict a correlation in the opposite sense, with the FeII generated deep inside the emitting clouds where only harder X-rays penetrate. 
- The fact that the soft X-ray energy indices correlate with independent properties (coredominance for RLQs and FeII equivalent widths for RQQs) argues for the wide range in the $\mathrm{X}$-ray slopes of both classes being real.

\section{Acknowledgements}

The authors would like to thank Beverley J. Wills for her comments. P. Shastri acknowledges support from the NSF grant AST-8714937 and NASA grant NAG 5-1700. M. Elvis, B.J. Wilkes and J. McDowell acknowledge support of the NASA grant NAGW5-2201 (SARP) and contracts NAS8-30751 (HEAO-2) and NAS5-30934 (RSDC).

\section{References}

Arnaud, K. A., et al., 1985, MNRAS 217, 105.

Blandford, R. D. and Konigl, A., 1979, ApJ 232, 34.

Biretta, J. A., Stern, C. P., and Harris, D. E. 1991, $A J$ 101, 1632

Boroson, T. A., 1989, ApJ 343, L9.

Browne, I. W. A. and Murphy, D. W., 1987, MNRAS 226, 601.

Burns, J. O., Feigelson, E. D. and Schreier, E. J., 1983, ApJ 273, 128.

Canizares, C. R. and White, J. L., 1989, ApJ 339, 27.

Comastri, A., Setti, G., Zamo:ani, G., Elvis, M., Giommi, P., Wilkes, B. J. and McDowell, J. C., 1992, ApJ 384, 62.

Cordova, F. A., Kartje, J., Rodriguez-Bell, T., Mason, K. O., and Harnden, F. R., 1989, in Extreme Ultraviolet Astronomy, eds. R. F. Malina and S. Bowyer, (New York: Pergamon Press), p. 30.

Elvis, M., Green, R. F. Bechtold, J., Schmidt, M., Neugebauer, G., Soifer, B. T., Matthews, K. and Fabbiano, G., 1986, ApJ 310, 291

Elvis, M., Wilkes, B. J., McDowell, J. C., Green, R. F. Bechtold, J., Willner, S. P., Cutri, R., Oey, M., S., and Polomski, E. 1992, ApJS in preparation

Fanti, R., Fanti, C., Schilizzi, R. T., Spencer, R. E. Nan Rendong, Parma, P., van Breugel, W. J. M. and Venturi, T., 1990, $A \& A$ 231, 333.

Fraix-Burnet, D. and Nieto, J.-L., 1988, $A \& A$ 198, 87.

Ghisellini, G., Maraschi L., and Treves, A., 1985, $A \& A$ 146, 204.

Harris, D. E. and Stern, C. P., 1987, ApJ 313,136.

Johnson, H. L., 1966, Ann. Rev. Astr. Ap., 4, 193.

Kapahi, V. K. and Saikia, D. J., 1982, J. Ap. \& Astr. 3, 465.

Kembhavi, A., Feigelson, E. D., and Singh, K. P., 1986, MNRAS 220, 51 .

Krolik, J. H. 1988, in Supermassive blackholes, ed. Kafatos, M., (Cambridge: Cambridge University Press), p. 279.

Lawrence, A., Saunders, W., Rowan-Robinson, M., Crawford, J., Ellis, R. S., Frenk, C. S., Efstathiou, G., and Kaiser, N., 1988, MNRAS 235, 261. 
Maraschi, L., 1992, in Variability of Blazars, eds. E. Valtaoja and M. Valtonen, (Cambridge: Cambridge University Press), p. 447.

Masnou, J.-L., Wilkes, B. J., Elvis, M., Mcdowell, J. C., and Arnaud, K. A., 1992, A $B A$ 253, 35.

Melia, F. and Konigl, A., 1989, in BL Lac Objects, eds., L. Maraschi, T. Maccacaro and M.-H. Ulrich, 1989, (Berlin: Springer- Verlag) p. 372.

Mushotzky, R. F., 1984, Adv. Sp. Res., 3, 157.

Orr, M. J. L. and Browne, I. W. A., 1982, MNRAS 200, 1067.

Netzer, H., Laor, A. and Gondhalekar, P. M. 1992, MNRAS 254, 15.

Peacock, J. A., Miller, L. and Mead, A. R. G., 1986, in IAU Symposium No. 119: Quasars eds., G. Swarup and V. K. Kapahi, (Dordrecht: Reidel), p. 103.

Petre, R., Mushotzky, R. F., Krolik, J. H. and Holt, S. S., 1984, ApJ 280, 499.

Pounds, K. A., Nandra, K, Stewart, G. C. George, I. M., and Fabian, A. C. 1990, Nature, 344, 132.

Shastri, P. 1991, MNRAS 249, 640.

Smith, E. P. and Heckman, T. M., 1990, ApJ 348, 38.

Turner, M. J. L., Williams, O. R., Saxton, R., Stewart, G. C., Courvoisier, T. J.-L., Ohashi, T., Makishima, K., Kii, T. and Inoue, H., 1989. in Proceedings of the 29rd ESLAB Symposium, Vol. 2, eds. J. Hunt and B. Batrick, (Noordwijk: ESA Publications Division), p. 769.

Turner, T. J. and Pounds, K. A. 1989, MNRAS 240, 833.

van Breugel, W., Miley, G., and Heckman, T. M., 1984. AJ 89, 5.

Wilkes, B. J. and Elvis, M., 1987 ApJ 323, 243.

Wilkes, B. J., Elvis, M. and McHardy, I., 1987 ApJ 321, L23.

Williams, O. R., Turner, M. J. L., Stewart, G. C., Saxton, R. D., Ohashi, T., Makishima, K., Kii, T., Inoue, H., Makino, F., Hayashida, K. and Koyama, K., 1992, ApJ 389, 157.

Wills, B. J., 1988, in Physics of Formation of FeII Lines Outside LTE, eds., R. Viotti, A. Vittani, and M. Freidjung, (Dordrecht: Reidel), p. 161.

Zamorani, G. et al., 1981, ApJ 245, 357.

Zheng, W. and O'Brien, P. T., 1990, ApJ 353, 433. 


\section{Figure Captions}

Fig. 1a. The distribution of the radio-loudness parameter $\left(R_{L_{t}}\right)$ for the quasars in our sample. (For the definition of radio-loudness see Section 2.)

Fig. 1b. The distributions of soft X-ray energy index $\alpha_{E}$ of the radio-loud (solid line) and radio-quiet (dashed line) quasars in our sample.

Fig. 2. The radio-loudness parameter $\left(R_{L_{t}}\right)$ (Section 2) plotted against the soft $\mathrm{X}$-ray energy index for the RLQs (filled squares) and RQQs (filled triangles) in the sample. The typical $1 \sigma$ error in the X-ray energy index is shown.

Fig. 3. For the RLQs, the soft X-ray energy index $\alpha_{E}$ plotted against the logarithm of the radio core-dominance parameter $R$ (Section 3 ).

Fig. 4a. The rest frame equivalent width of the FeII $\lambda 4570$ multiplet plotted against the soft X-ray energy index $\alpha_{E}$. Separately shown are RQQs (filled triangles), CDQs (crossed squares) and LDQs (empty squares). The single filled square is the Ginga measurement for 1416-129, whose IPC measurement has large errors. The dashed line represents a simple regression on the RQQ data to allow comparison with Figure $4 \mathrm{~b}$.

Fig. 4b. The same plot as in 4(a) but for the data from Zheng \& O'Brien (1990). The symbols are as in 4(a). The Compact Steep Spectrum (CSS) object is $0134+329$ (3C48). The regression line from Fig $4 \mathrm{a}$ is shown for comparison. 
Table 1: Radio-quiet Quasars

\begin{tabular}{|c|c|c|c|c|c|c|c|c|c|}
\hline Source & $\begin{array}{l}\text { Other } \\
\text { Name }\end{array}$ & Redshift & $\begin{array}{l}f_{B}^{a} \\
\mathrm{mJy}\end{array}$ & $\mathrm{R}_{L_{t}}$ & $\begin{array}{c}\alpha_{E} \\
( \pm 1 \sigma)\end{array}$ & $\begin{array}{l}\text { Ref.c }^{c} \\
\left(\alpha_{E}\right) \\
\end{array}$ & $\begin{array}{c}\mathrm{W}_{\lambda}^{b}(\mathrm{FeII}) \\
(\AA) \\
\end{array}$ & $\begin{array}{l}\text { Ref. }^{c} \\
\left(\mathrm{~W}_{\lambda}\right) \\
\end{array}$ & $\begin{array}{c}\text { FWHM }(\mathrm{H} \beta)^{d} \\
\mathrm{~km} \mathrm{~s}^{-1}\end{array}$ \\
\hline $0026+129$ & PG & 0.142 & 2.33 & 0.34 & $0.88_{-0.05}^{+0.05}$ & 4 & & & \\
\hline $0054+144$ & PHL909 & C.171 & 1.02 & -0.01 & $0.41_{-0.07}^{+0.07}$ & 4 & & & \\
\hline $0205+024$ & $\mathrm{NAB}$ & 0.155 & 2.25 & -0.18 & $1.2_{-0.07}^{+0.07}$ & 4 & & & \\
\hline $0844+349^{f}$ & PG & 0.064 & 11.15 & -1.56 & $1.6_{-0.2}^{+0.7}$ & 2 & 46 & 1,2 & 3380 \\
\hline $0923+129^{f}$ & PG & 0.028 & 4.74 & 0.32 & $0.7_{-0.2}^{+0.3}$ & 2 & 29 & 2 & 1840 \\
\hline $1116+215$ & PG & 0.177 & 3.80 & -0.13 & $1.0_{-0.2}^{+0.3}$ & 3 & 15 & 1 & 4290 \\
\hline $1202+281$ & GQComae & 0.165 & 2.33 & -0.45 & $1.1_{-0.4}^{+0.2}$ & 3,6 & & & \\
\hline $1211+143$ & PG & 0.085 & 6.24 & 1.40 & $1.8_{-0.2}^{+0.3}$ & 3 & $32^{e}$ & 1,2 & 3000 \\
\hline $1219+755$ & Mkn205 & 0.070 & 2.46 & -0.31 & $0.78_{-0.03}^{+0.03}$ & 4 & & & \\
\hline $1229+204$ & Ton 1542 & 0.064 & 2.63 & -0.59 & $1.5_{-0.1}^{+0.2}$ & 2 & 45 & 2 & 3170 \\
\hline $1244+026$ & PG & 0.048 & 1.54 & -0.27 & $1.6_{-0.5}^{+0.7}$ & 3,6 & & & \\
\hline $1304+346$ & AB133 & 0.189 & 0.50 & -0.58 & $1.0_{-0.2}^{+0.6}$ & 1 & & & \\
\hline $1307+085$ & PG & 0.158 & 3.43 & -0.99 & $0.9_{-0.2}^{+0.5}$ & 3,6 & $<8$ & 1 & 5570 \\
\hline $1407+265$ & PG & 0.944 & 2.27 & 0.54 & $1.2_{-0.2}^{+0.9}$ & 3 & & & \\
\hline $1416-129$ & PG & 0.129 & 3.07 & 0.07 & $0.9_{-0.5}^{+0.5}$ & 3 & $<3$ & 1 & 4640 \\
\hline $1426+015$ & PG & 0.086 & 3.66 & -0.48 & $1.2_{-0.2}^{+0.2}$ & 3 & $>20$ & 1 & 5540 \\
\hline $1501+106$ & Mkn841 & 0.036 & 7.04 & -0.67 & $0.93_{-0.04}^{+0.04}$ & 4 & $26^{e}$ & 1 & 5360 \\
\hline $1613+658$ & PG & 0.129 & 2.42 & 0.10 & $1.1_{-0.3}^{+0.2}$ & 3 & 16 & 1 & 7050 \\
\hline $1803+676$ & KAZ102 & 0.136 & 1.70 & -0.69 & $-0.2_{-0.3}^{+0.4}$ & 3 & $<0.2$ & 1,2 & 4810 \\
\hline $2130+099$ & PG,IIZw136 & 0.061 & 4.78 & -0.37 & $0.81_{-0.07}^{+0.07}$ & 4 & & & \\
\hline $2251-178$ & MR & 0.068 & 4.48 & -0.15 & $0.3_{-0.6}^{+0.5}$ & 1 & & & \\
\hline
\end{tabular}

a: $f_{B}$ is calculated from $B$ using $\mathrm{m}_{B}(0)=4440 \mathrm{Jy}$ (Johnson 1966). When $B$ magnitudes were not available, $V$ magnitudes were used.

b: Equivalent width of Fell $\lambda 4570$ in the quasar's rest frame (uncertainites are $\sim \pm 10 \%$ ).

c: References: (1) this paper; (2) Wilkes, Elvis and McHardy 1987; (3) Wilkes and Elvis 1987;

(4) Masnou et al. 1992; (5) Blvis et al. 1992; (6) Elvis et al. 1986.

d: Full width at half-maximum of $\mathrm{H} \beta$ in the observed frame.

e: HeII $\lambda 4686$ excluded from rneasurement.

f: Errors on $\alpha_{E}$ are scaled to $1 \sigma$ from the $90 \%$ errors given in Wilkes, Elvis and McHardy (1987). 
Table 2: Radio-loud Quasars

\begin{tabular}{|c|c|c|c|c|c|c|c|c|c|c|}
\hline Source & $\begin{array}{l}\text { Other } \\
\text { Name }\end{array}$ & Redshift & $\begin{array}{c}\mathrm{f}_{\boldsymbol{B}} \\
\mathrm{mJy}\end{array}$ & $\mathrm{R}_{L_{t}}$ & $\mathrm{R}$ & $\begin{array}{l}\alpha_{E} \\
( \pm 1 \sigma)\end{array}$ & $\begin{array}{l}\text { Ref.c }^{c} \\
\left(\alpha_{E}\right)\end{array}$ & $\begin{array}{l}W_{\lambda}^{b} \\
(\AA) \\
\end{array}$ & $\begin{array}{l}\text { Ref. }^{c} \\
\left(W_{\lambda}\right)\end{array}$ & $\begin{array}{l}\text { FWHM }^{d} \\
\mathrm{~km} \mathrm{~s}^{-1}\end{array}$ \\
\hline $0007+106$ & III $\mathrm{Zw}_{2}$ & 0.089 & 1.83 & 2.24 & 0.9 & $0.4_{-0.3}^{+0.9}$ & 3 & 5 & 2 & 4700 \\
\hline $0133+207$ & $3 \mathrm{C} 47$ & 0.425 & 0.24 & 3.65 & 0.07 & $0.9_{-0.3}^{+0.3}$ & 3 & & & \\
\hline $0134+329$ & $3 C 48$ & 0.367 & 1.40 & 3.61 & CSS & $0.7_{-0.4}^{+0.4}$ & 3 & & & \\
\hline $0312-770$ & PKS & 0.223 & 1.39 & 2.60 & 10.9 & $0.1_{-0.2}^{+0.6}$ & 3 & 18 & 2 & 3340 \\
\hline $0637-752$ & PKS & 0.656 & 1.64 & 3.53 & $>10$ & $0.5_{-0.1}^{+0.1}$ & 4 & & & \\
\hline $0837-120$ & $3 \mathrm{C} 206, \mathrm{PKS}$ & 0.198 & 2.16 & 2.58 & 0.3 & $0.6_{-0.3}^{+0.3}$ & 5 & $<8$ & 1 & 4485 \\
\hline $0903+169$ & 3 C 215 & 0.411 & 0.18 & 3.36 & 0.05 & $0.0_{-0.2}^{+0.6}$ & 3 & & & \\
\hline $0923+392$ & $4 C 39.25$ & 0.699 & 0.30 & 4.39 & 36 & $0.4_{-0.1}^{+0.2}$ & 3 & & & \\
\hline $1020-103$ & PKS & 0.197 & 1.40 & 2.44 & 5.5 & $0.8_{-1.6}^{+0.7}$ & 1 & & & \\
\hline $1028+313$ & B2 & 0.177 & 0.66 & 2.38 & 1.3 & $0.62_{-0.06}^{+0.06}$ & 4 & $<3$ & 1 & 4280 \\
\hline $1100+772$ & 3C 249.1 & 0.311 & 2.33 & 2.52 & 0.1 & $1.0_{-0.5}^{+0.3}$ & 3 & & & \\
\hline $1111+408$ & $3 \mathrm{C} 254$ & 0.734 & 0.25 & 3.50 & 0.02 & $1.0_{-0.3}^{+0.9}$ & 1 & & & \\
\hline $1128+315$ & B2 & 0.289 & 1.02 & 2.39 & 9.6 & $0.7_{-0.4}^{+0.5}$ & 1 & & & \\
\hline $1137+660$ & $3 C 263$ & 0.656 & 1.12 & 2.97 & 0.2 & $0.7_{-0.1}^{+0.4}$ & 3 & & & \\
\hline $1146-037$ & PKS & 0.341 & 0.73 & 2.37 & 1.4 & $0.3_{-0.2}^{+0.5}$ & 3 & 3 & 2 & 4300 \\
\hline $1217+023$ & PKS & 0.240 & 1.07 & 2.64 & 2.0 & $0.5_{-0.1}^{+0.3}$ & 3 & & & \\
\hline $1226+023$ & $3 \mathrm{C} 273$ & 0.158 & 26.27 & 3.12 & 5.5 & $0.47_{-0.03}^{+0.03}$ & 4 & 15 & 1,2 & 4300 \\
\hline $1253-055$ & 3 C 279 & 0.538 & 0.28 & 4.58 & 7.8 & $0.6_{-0.2}^{+0.1}$ & 3 & & & \\
\hline $1545+210$ & $3 \mathrm{C} 323.1$ & 0.266 & 0.85 & 3.04 & 0.05 & $0.8_{-0.5}^{+0.3}$ & 3,6 & & & \\
\hline $1635+119$ & MC & 0.146 & 0.72 & 2.05 & 0.3 & $0.9_{-0.6}^{+1.0}$ & 3 & & & \\
\hline $1704+608$ & 3C351 & 0.371 & 3.04 & 2.60 & 0.02 & $0.1_{-0.5}^{+0.9}$ & 5 & $3^{e}$ & 1 & 2420 \\
\hline $1721+343$ & $4 \mathrm{C} 34.47$ & 0.206 & 1.12 & 2.76 & 1.2 & $0.5_{-0.3}^{+0.4}$ & 3 & & & \\
\hline $2128-123$ & PKS & 0.501 & 2.49 & 2.92 & $>20$ & $0.5_{-0.3}^{+0.8}$ & 1 & & & \\
\hline $2135-147$ & PKS & 0.200 & 1.77 & 2.99 & 0.09 & $0.73_{-0.05}^{+0.05}$ & 4 & & & \\
\hline
\end{tabular}

a: $f_{B}$ is calculated from $B$ using $m_{B}(0)=4440 \mathrm{Jy}$ (Johnson 1966). When $B$ magnitudes were not available, $\mathrm{V}$ magnitudes were used.

b: Equivalent width of FeII $\lambda 4570$ in the quasar's rest frame (uncertainites are $\sim \pm 10 \%$ ).

c: References: (1) this paper; (2) Wilkes, Elvis and McHardy 1987; (3) Wilkes and Elvis 1987;

(4) Masnou et al. 1992; (5) Elvis et al. 1992; (6) Elvis et al. 1986.

$\mathrm{d}$ : Full width at half-maximum of $\mathrm{H} \beta$ in the observed frame.

e: HeII $\lambda 4686$ excluded from measurement. 
Authors' Addresses

M. Elvis, J. C. McDowell and B.J. Wilkes: Harvard-Smithsonian Center for Astrophysics, 60 Garden Street, Cambridge MA 02138.

P. Shastri: Astronomy Department, University of California, Berkeley CA 94720. 


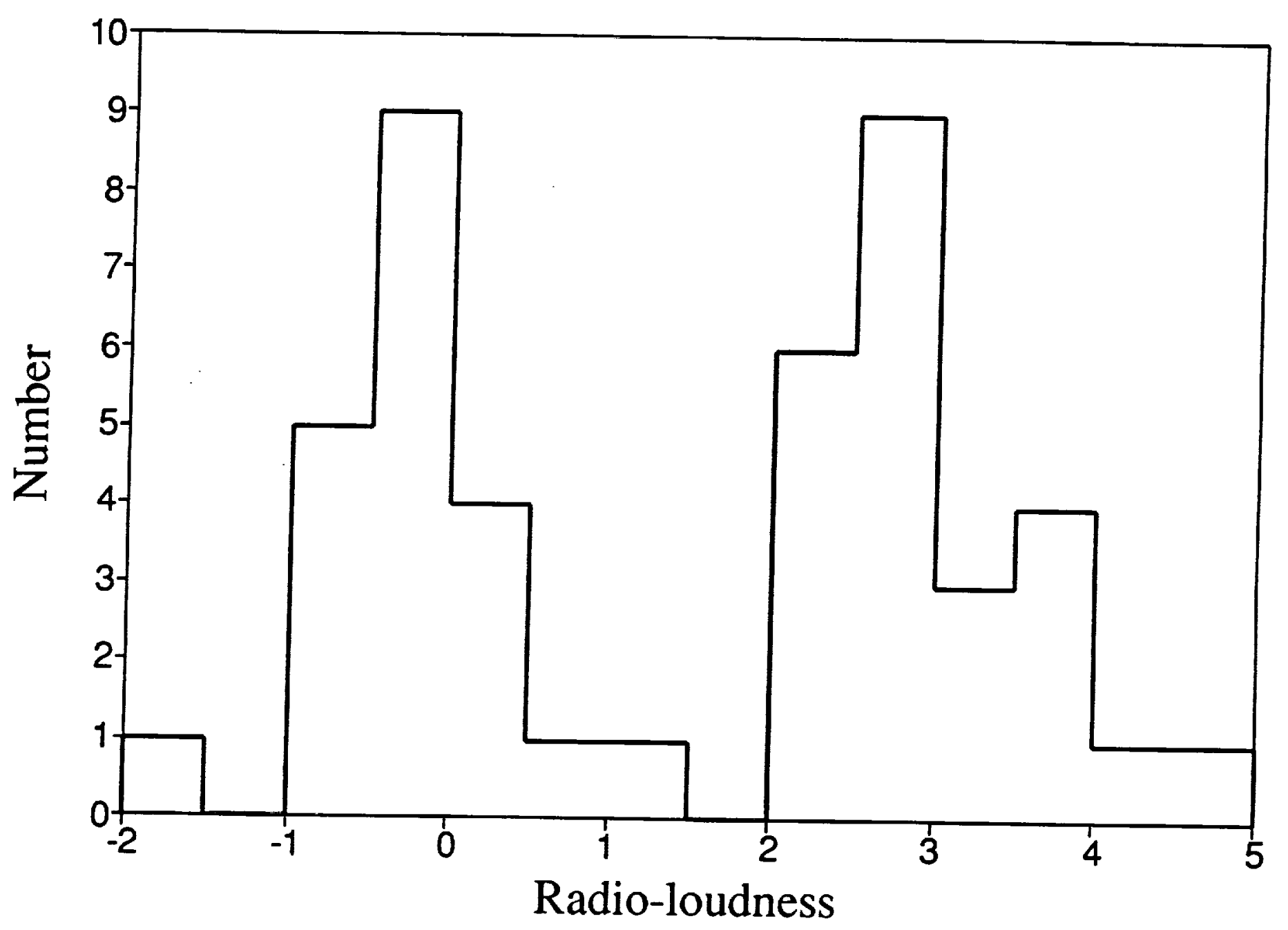

Figure 1. 


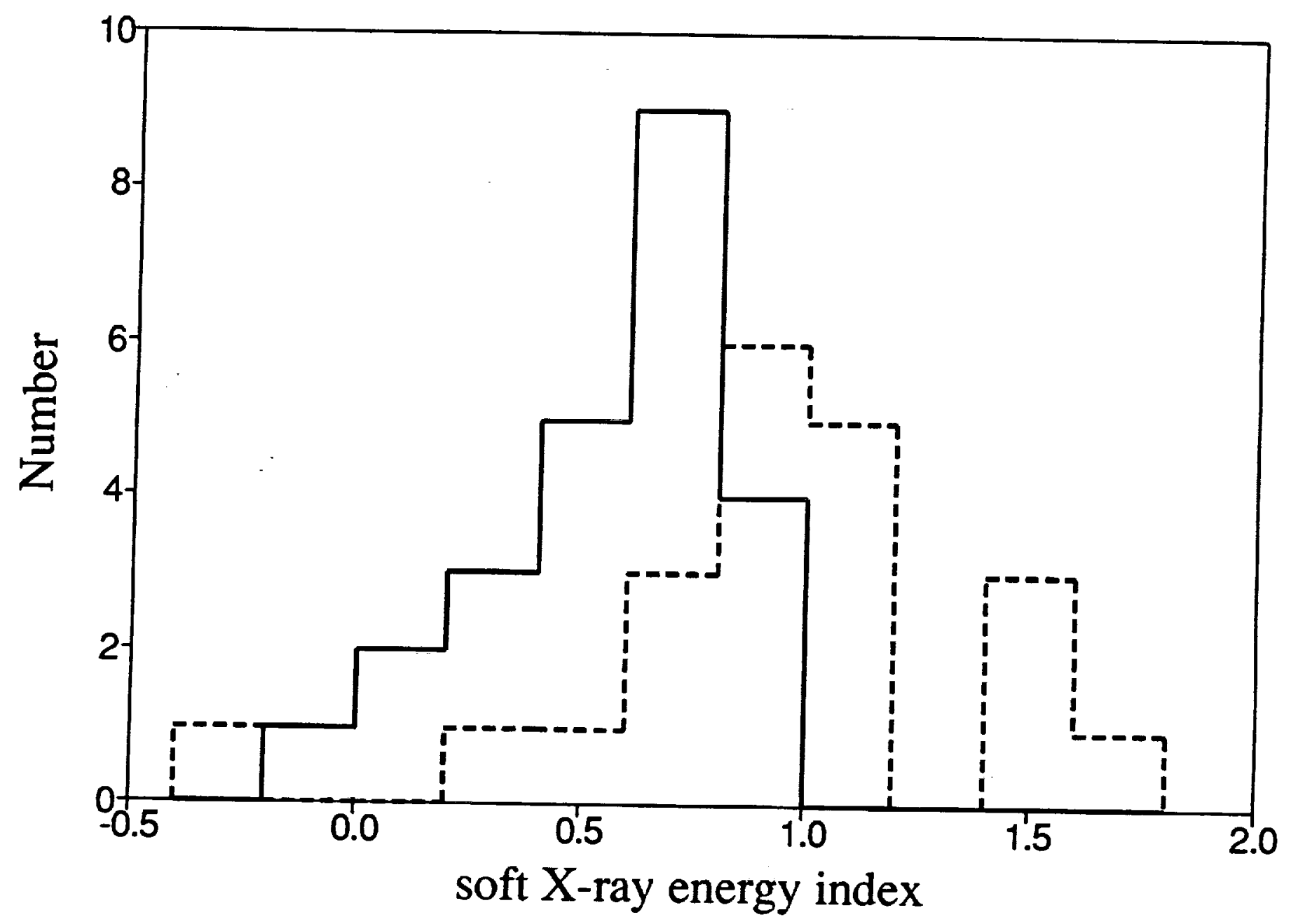

Figure Ib 


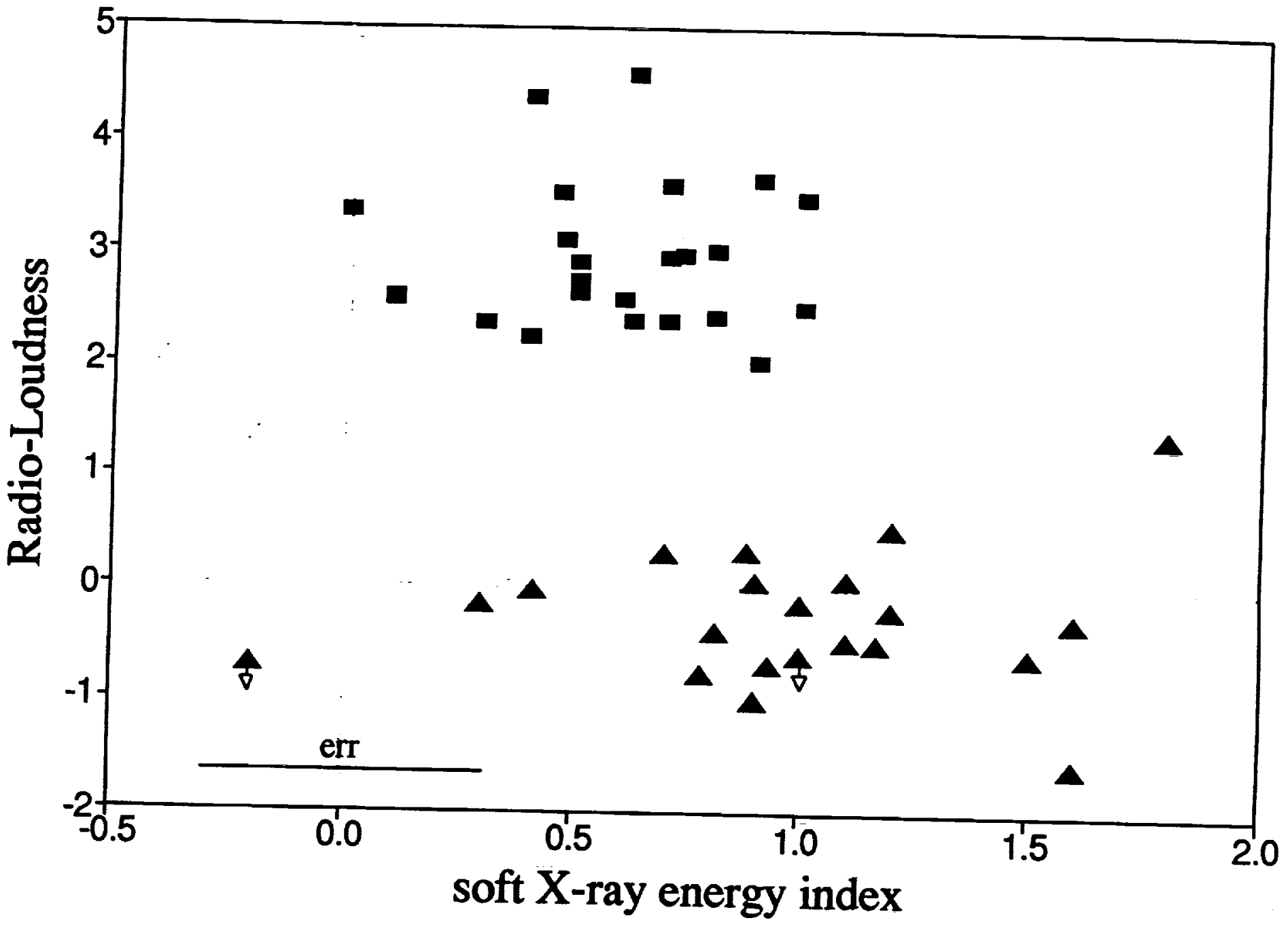

Figure 2 


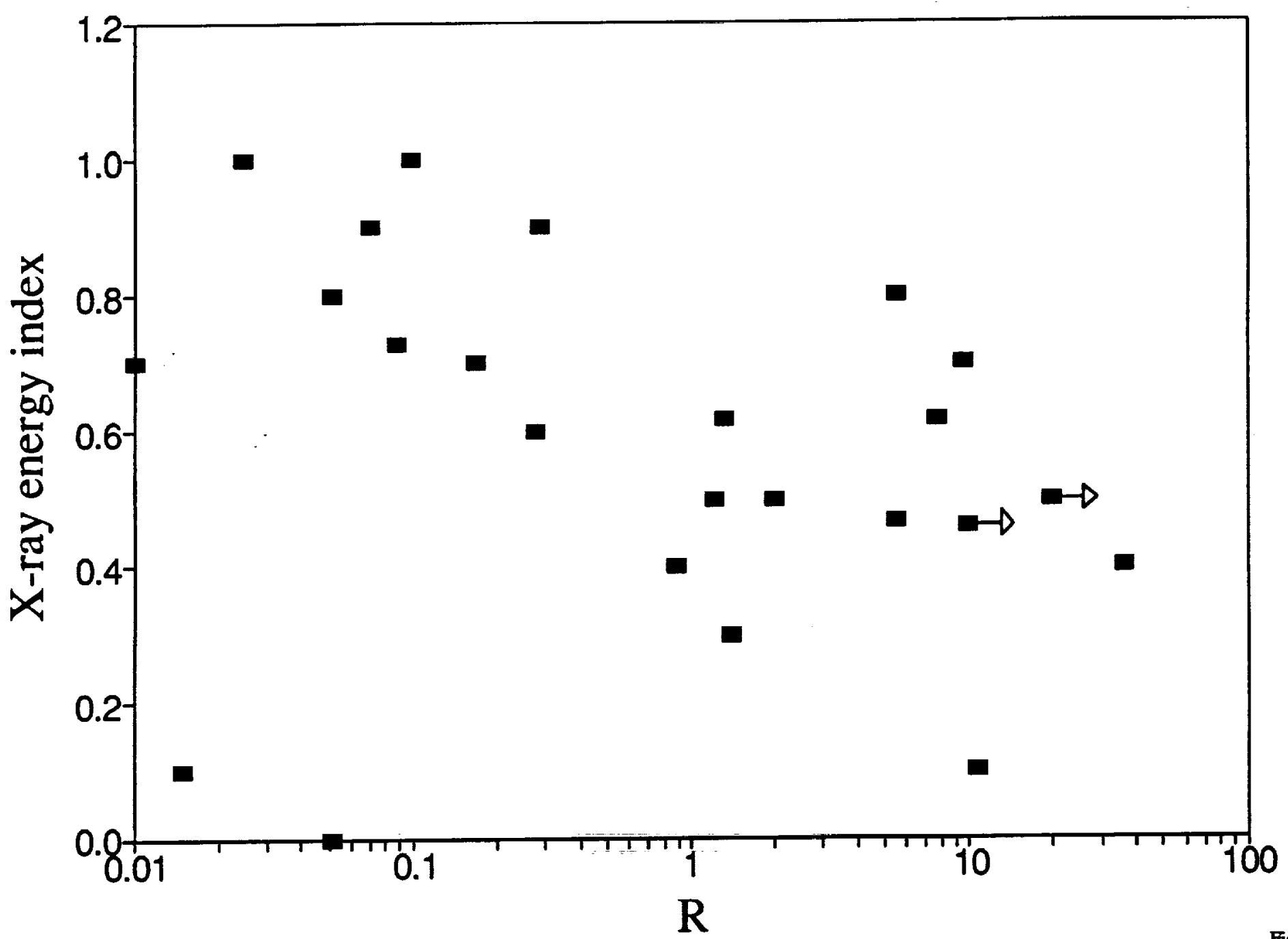

Figure 3 


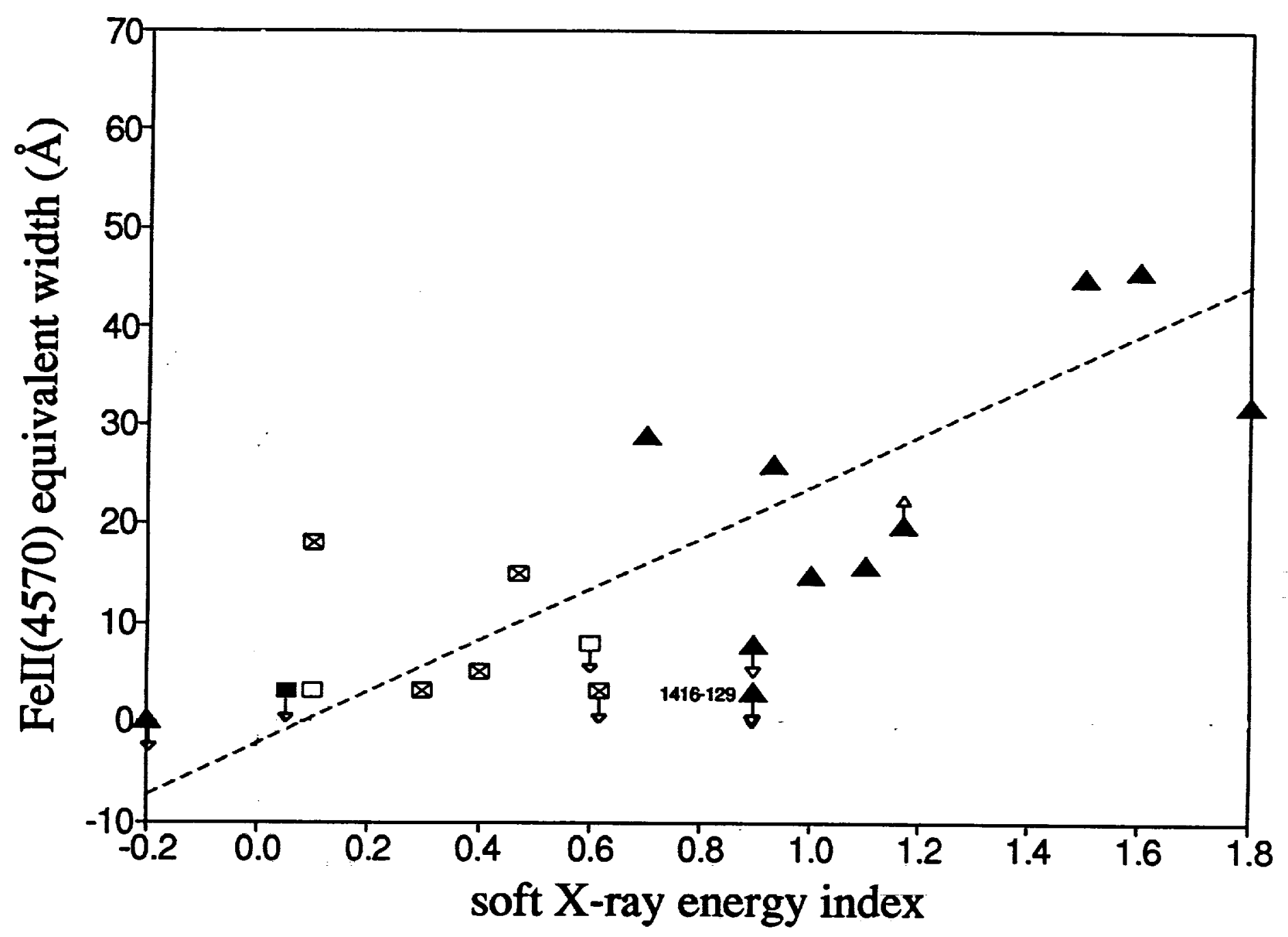

Figure 4a 


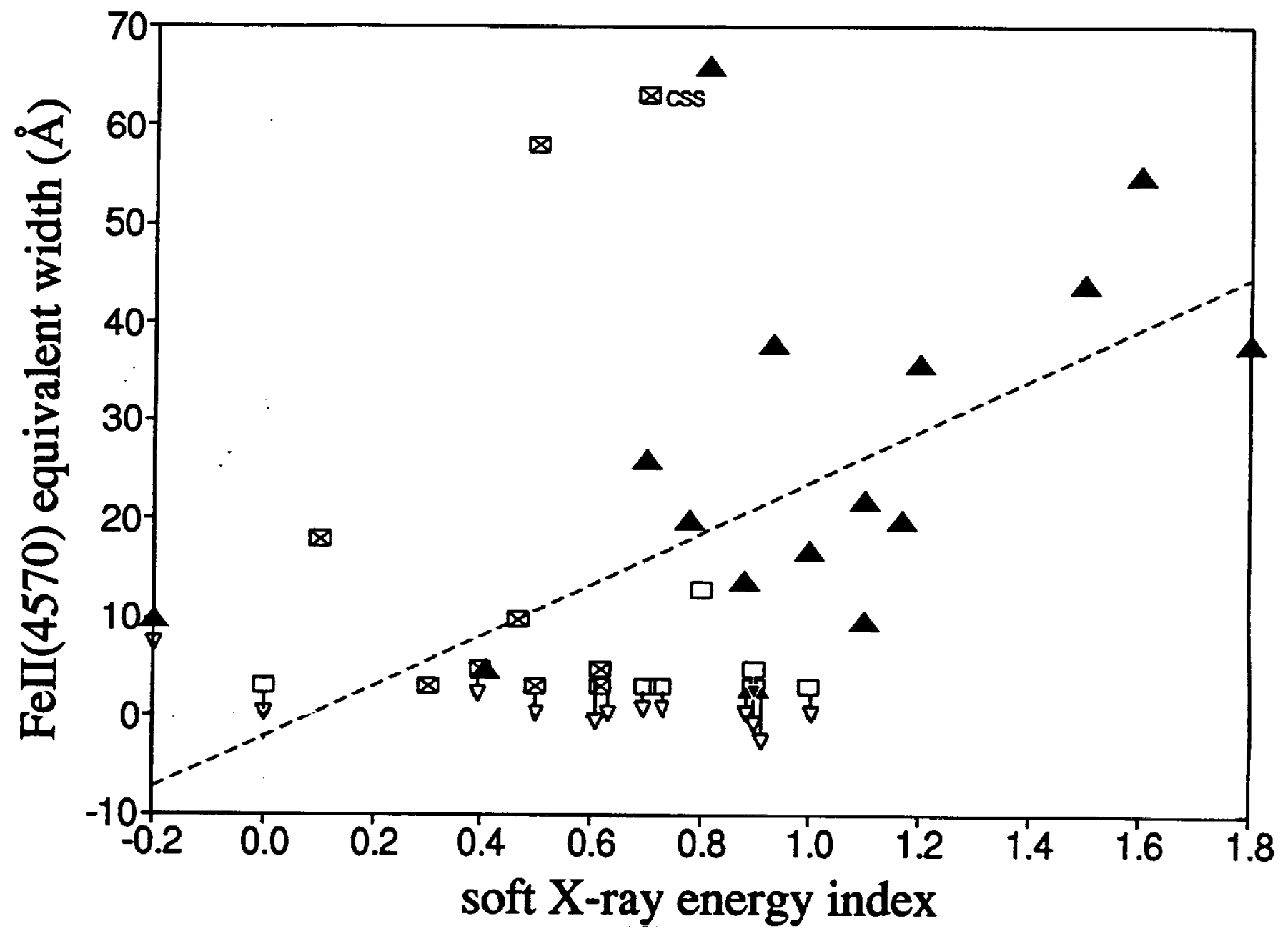

Figure $4 b$ 
. 